\title{
Stable rotating dipole solitons in nonlocal optical media
}

Lopez-Aguayo, Servando; Desyatnikov, Anton S.; Kivshar, Yuri S.; Skupin, Stefan; Krolikowski, Wieslaw; Bang, Ole

\section{Published in:}

Optics Letters

Link to article, DOI:

10.1364/OL.31.001100

Publication date:

2006

Document Version

Publisher's PDF, also known as Version of record

Link back to DTU Orbit

Citation (APA):

Lopez-Aguayo, S., Desyatnikov, A. S., Kivshar, Y. S., Skupin, S., Krolikowski, W., \& Bang, O. (2006). Stable rotating dipole solitons in nonlocal optical media. Optics Letters, 31(8), 1100-1102.

https://doi.org/10.1364/OL.31.001100

\section{General rights}

Copyright and moral rights for the publications made accessible in the public portal are retained by the authors and/or other copyright owners and it is a condition of accessing publications that users recognise and abide by the legal requirements associated with these rights.

- Users may download and print one copy of any publication from the public portal for the purpose of private study or research.

- You may not further distribute the material or use it for any profit-making activity or commercial gain

- You may freely distribute the URL identifying the publication in the public portal 


\title{
Stable rotating dipole solitons in nonlocal optical media
}

\author{
Servando Lopez-Aguayo, Anton S. Desyatnikov, and Yuri S. Kivshar \\ Nonlinear Physics Center, Research School of Physical Sciences and Engineering, Australian National University, \\ Canberra, ACT 0200, Australia \\ Stefan Skupin and Wieslaw Krolikowski \\ Laser Physics Center, Research School of Physical Sciences and Engineering, Australian National University, \\ Canberra, ACT 0200, Australia
}

\begin{abstract}
Ole Bang
$C O M \bullet D T U$ Department of Communications, Optics \& Materials, Technical University of Denmark, DK-2800 Kongens, Lyngby, Denmark
\end{abstract}

\begin{abstract}
Received December 20, 2005; revised January 15, 2006; accepted January 17, 2006; posted January 23, 2006 (Doc. ID 66786)
We reveal that nonlocality can provide a simple physical mechanism for stabilization of multihump optical solitons and present what we believe to be the first example of stable rotating dipole solitons and soliton spiraling, which are known to be unstable in all types of realistic nonlinear media with a local response. (C) 2006 Optical Society of America OCIS codes: $190.0190,190.4420$.
\end{abstract}

Recently increased interest in the study of selftrapped optical beams in nonlocal nonlinear media is explained by experimental observations of nonlocal spatial solitons in liquid crystals ${ }^{1,2}$ and lead glasses ${ }^{3}$ as well as a number of interesting theoretical predictions, ${ }^{4-7}$ including stabilization of vortex solitons against symmetry breaking instability in media with a nonlocal optical response. ${ }^{8,9}$ Many of the predicted and demonstrated properties of nonlocal nonlinear models suggest that in such optical media we should expect stabilization of many different types of nonlinear structures such as necklaces, ${ }^{10}$ soliton clusters, ${ }^{11}$ and recently introduced broader classes of modulated optical vortices. ${ }^{12}$

One of the simplest multihump solitons predicted in nonlinear optics is a dipolelike structure composed of two interacting fundamental beams with opposite phases that undergo angular rotation during propagation. ${ }^{13,14}$ Such a localized structure can be viewed as a special type of strongly modulated singlecharge vortex soliton or azimuthon, and it can also be linked to the problem of soliton spiraling, as discussed, e.g., in Sects. 6.1 of Ref. 13. However, despite their broad applicability and deep physical context, rotating scalar dipoles are known to be always unstable in all types of realistic optical media with a local response. ${ }^{13,14}$ Stabilization can be achieved by incoherent interaction with a fundamental beam. ${ }^{14,15}$

In this Letter we study the effect of the nonlocal response of an optical medium on the stability of rotating dipole solitons. We demonstrate that such solitons can be stabilized when the nonlocality parameter exceeds some threshold value that decreases with angular momentum and angular velocity, i.e., the dipoles that are rotating faster are more robust. We describe a continuous family of two-lobe localized structures (or azimuthons ${ }^{12}$ ) that extend from nonrotating dipole solitons to radially symmetric vortex solitons by continuous azimuthal transformations.

We consider the propagation of paraxial optical beams in a nonlinear medium described by the nonlinear Schrödinger equation ${ }^{13}$ for the scalar field envelope $E$ :

$$
i \partial_{z} E+\nabla^{2} E+n(I, \vec{r}) E=0,
$$

where $z$ and $\vec{r}$ stand for the propagation and transverse coordinates, respectively. We assume that the nonlinear refractive index $n$ depends on the intensity $I \equiv|E|^{2}$ via the following nonlocal relation:

$$
n(I, \vec{r})=\int R(|\vec{r}-\vec{\rho}|) I(\vec{\rho}) d \vec{\rho},
$$

where the response function $R(r)$ is determined by the specific physical process responsible for the medium nonlinearity. Here we consider a Gaussian nonlocal response

$$
R(r)=\sigma^{-2} \pi^{-1} \exp \left(-r^{2} / \sigma^{2}\right),
$$

where $\sigma$ measures the degree of nonlocality. In the following we use the scaling $\vec{r}=\vec{r}^{\prime} \sigma, z=z^{\prime} \sigma^{2}, E=E^{\prime} / \sigma$, and omit primes, because physically relevant beam power $P \equiv \int|E|^{2} \mathrm{~d} \vec{r}=\int\left|E^{\prime}\right|^{2} \mathrm{~d} \vec{r}^{\prime}$ does not depend on $\sigma$.

Multihump localized solutions of this model can be found numerically by two-dimensional relaxation methods. ${ }^{16}$ However, this approach represents a major difficulty for the complex envelopes with singular phase profiles due to a poor convergence. Therefore, here we employ an approximate but much simpler variational method ${ }^{17}$ allowing a reasonably good approximation. ${ }^{12}$ To this end, we introduce the following ansatz: 


$$
E=\sqrt{\pi} U(r)[\cos (m \varphi)+i p \sin (m \varphi)] \exp (i k z),
$$

where $\varphi$ is the azimuthal angle and the parameter $p$ $(0 \leqslant p \leqslant 1)$ determines the modulation depth (contrast) of the beam intensity, $n=1-p^{2}$. Substituting this ansatz into the averaged Lagrangian ${ }^{17}$ associated with Eq. (1), we obtain the equation for the radial amplitude $U(r)$,

$$
-k U+U_{r r}+r^{-1} U_{r}-m^{2} r^{-2} U+U N\left(U^{2}, r\right)=0,
$$

where the nonlinear term is given by the expression

$$
\begin{aligned}
N\left(U^{2}, r\right)= & \pi\left(1+p^{2}\right) \mathrm{e}^{-r^{2}} \int_{0}^{\infty} \rho \mathrm{d} \rho \mathrm{e}^{-\rho^{2}} U^{2}(\rho) \\
& \times\left\{I_{0}(2 r \rho)+\frac{1}{2}\left(\frac{1-p^{2}}{1+p^{2}}\right)^{2} I_{2 m}(2 r \rho)\right\},
\end{aligned}
$$

and $I_{j}$ is the modified Bessel function of the first kind. In the limit $p \rightarrow 1$, the function (4) becomes radially symmetric, and the potential (6) corresponds to a vortex soliton. On the other hand, for $p \rightarrow 0$, this solution describes scalar multipole solitons ${ }^{14}$ (or optical necklaces) known to be radially unstable in local media.

Applying to Eq. (5) the numerical shooting technique and an iteration procedure, we find the stationary localized solutions with different values of $p$ and $k$. Our results are summarized in Fig. 1. Figures 1(a) and $1(\mathrm{~b})$ show the radial envelopes of the localized modes (solid curves) and the corresponding profiles of the effective refractive index (dashed curves) for the dipole $(p=0)$ and vortex $(p=1)$ solitons in the case of low [Fig. 1(a)] and high [Fig. 1(b)] degrees of nonlocality. Figure 1(c) shows the dependence of the soliton power $P=\pi\left(1+p^{2}\right) \int_{0}^{\infty} U^{2} r \mathrm{~d} r$ on the propagation con-
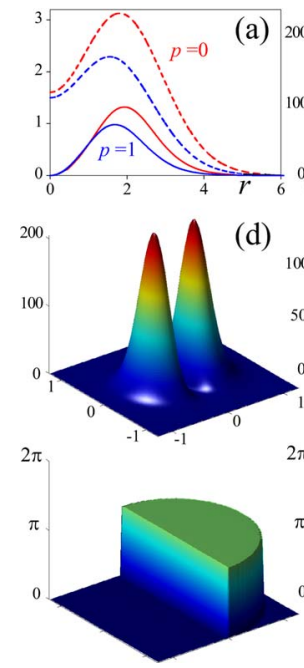

(d)

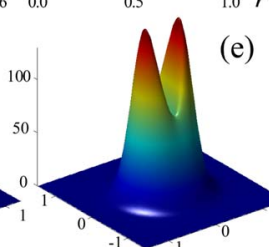

(e)
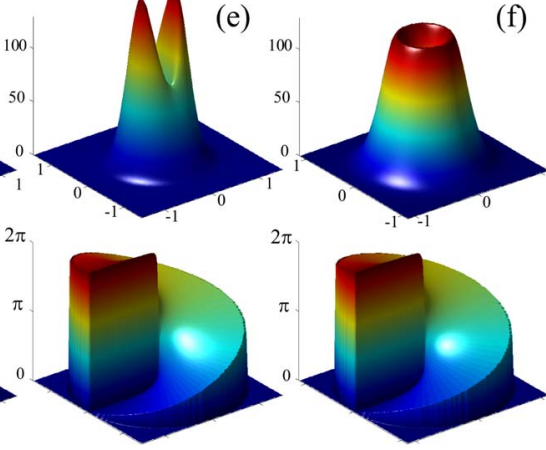

$2 \pi$

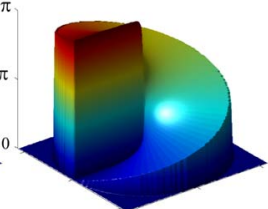

Fig. 1. (Color online) Beam radial intensity $U^{2}(r)$ (solid curves) and effective nonlinear potential $N(U, r)$ (dashed curves) for the dipole soliton ( $p=0$, red) and vortex soliton ( $p=1$, blue) for (a) relatively low nonlocality with $k=1$ and (b) high nonlocality with $k=70$. (c) Power versus propagation constant $k$ for the dipole solitons; several lines for different $p$ coincide. (d)-(f) Envelopes of the (top) intensity and (bottom) phase for three characteristic cases with $k$ $=70$ and (d) $p=0$, (e) $p=2^{-1 / 2}$, (f) $p=1$. stant $k$, for several values of modulation parameter $p$. Figures 1(d)-1(f) present the intensity (top) and phase (bottom) distributions for the dipole, azimuthon, and vortex solitons, respectively.

Having a nontrivial phase structure, the azimuthons carry a nonzero angular momentum. Using the ansatz Eq. (4), we find that the beam orbital angular momentum, $M=\operatorname{Im} \int E^{*} \partial_{\varphi} E \mathrm{~d} \vec{r}$, normalized to its power $P$, defines the beam (fractional) spin, $M / P$ $=2 p /\left(1+p^{2}\right)$.

These results show the effect of the modulation depth (determined by $p$ ) on the soliton intensity, and they demonstrate that a transition from a dipole to a radially symmetric vortex soliton can be achieved by continuous azimuthal transformations. Therefore, the two-peak azimuthon solutions described above provide a link between two spiraling solitons with opposite phase and radially symmetric spatially localized optical vortices.

To study the stability of the stationary solutions we solve Eq. (1) numerically by the split-step beam propagation method with a fast Fourier transform. As initial conditions, we take the stationary solutions perturbed by $10-20 \%$ of noise. Note that, because we measure transverse scale in units of characteristic nonlocality length $\sigma$, the soliton propagation constant scales as $k \sim \sigma^{2}$, and we refer to cases with large $k$ as the strongly nonlocal regime. While the absolute values of the soliton period $\sim \pi / k$ as well as corresponding propagation distances became small in this regime, we always propagate solitons over distances of hundreds of soliton periods.

Figure 2 presents typical propagation scenarios. When the nonlocality is weak the dipole breaks into two mutually repelling filaments [Fig. 2(a)], and this behavior is reminiscent of the azimuthal instability of vortices in local media. ${ }^{14}$ For subcritical nonlocality [Fig. 2(b)] the azimuthon initially breaks up into two filaments that are subsequently forced by the nonlocality-induced potential to collide and merge into a single fundamental soliton. Stable propagation of the rotating azimuthon is observed when nonlocality exceeds a critical value [Fig. 2(c)]. This corresponds to a balance of the effective nonlocalitymediated attractive force and centrifugal repulsive force. The domain of stability on the plane $(p, k)$ is shown in Fig. 2(d), and the stability threshold decreases in the limit of the radially symmetric vortices $(p \rightarrow 1)$. For the stable rotating dipoles we obtain angular velocity $\Omega$ by numerical averaging, and Fig. 2 (e) shows the dependence of the azimuthon angular velocity on the modulation parameter $p$. The velocity increases monotonically with $p$ and depends only weakly on the power (two curves are for $k=60$ and $k=80$ ). For a vortex soliton with $p=1$ the rotation velocity is a free parameter.

We have tested the stability of rotating dipole solitons with respect to strong perturbations that lead to large-amplitude oscillations, as shown in Figs. 3(a) and 3(b). Similar studies have been carried out for spatial solitons with higher azimuthal indices $m>1$ (not shown). We obtained somewhat surprising results by testing the stability of double-ring vortex 

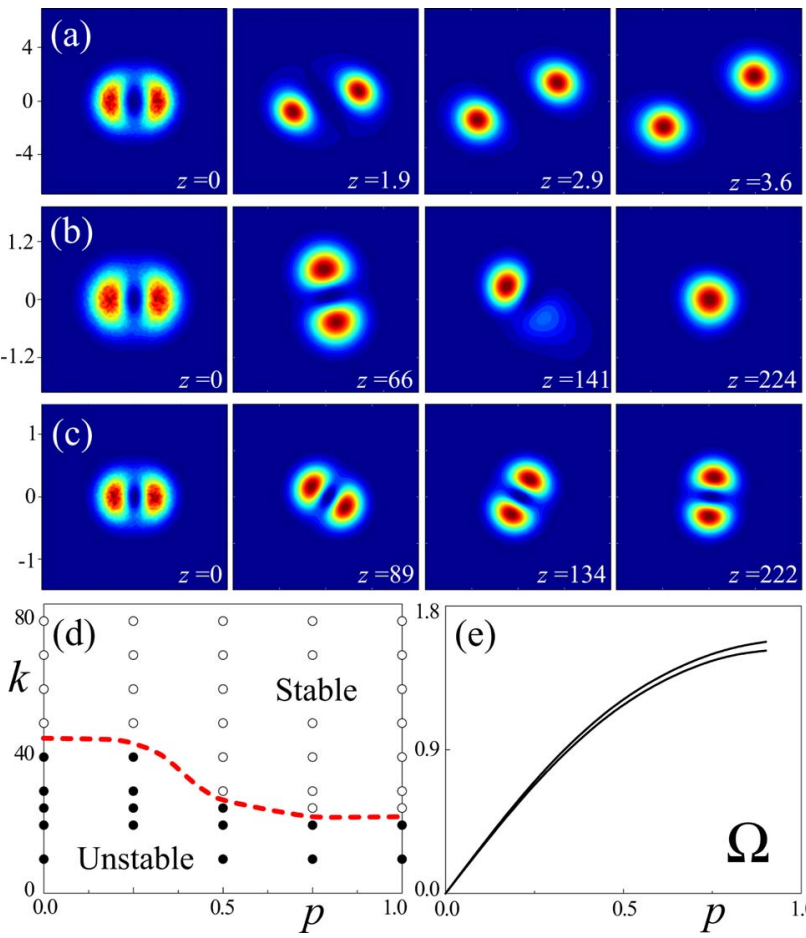

(e)

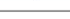

$=22$

\section{$\sim$}
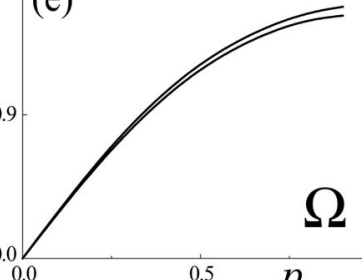

$\Omega$

Fig. 2. (Color online) Two different instability scenarios for rotating dipole solitons with $p=0.5$ for (a) $k=1$ and (b) $k=20$. (c) Stable rotation for $p=0.5$ and $k=100$. In all three cases the initial noise is $10 \%$. (d) Stability domain of dipole solitons in the $(p, k)$ space. (e) Numerically found relation between the angular velocity $\Omega$ of a dipole soliton and its contrast parameter $p$.

(a)

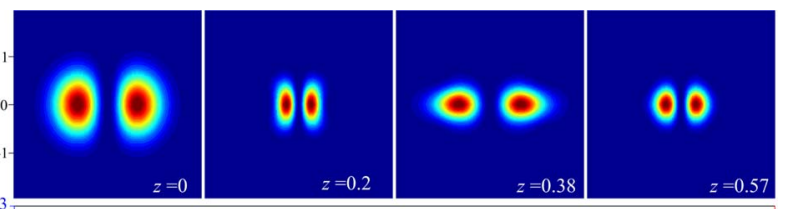

(b)

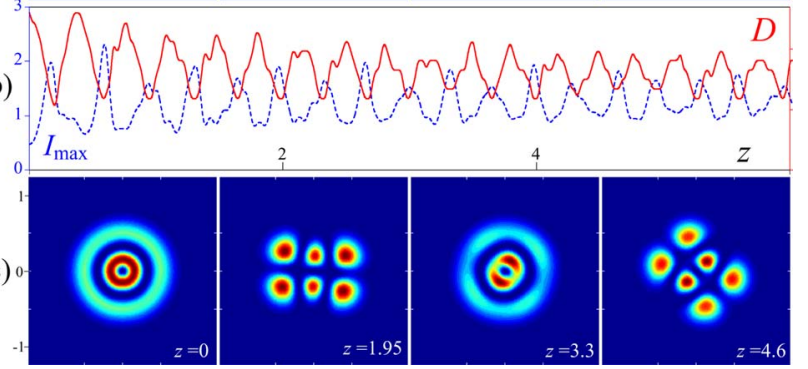

Fig. 3. (Color online) (a), (b) Evolution of a dipole soliton $(k=60, p=0)$ excited by a strong initial pertubation. Persistent oscillations of both the mode amplitude $I_{\max }$ (dashed curve) and the diameter $D$ (a distance between two peaks, solid curve) are observed; these oscillations decay slowly during propagation. (c) Example of the soliton revivals observed in the instability-induced evolution of double-ring single-charge vortex solitons with $k=100$.

solitons $(p=1)$ with $m=1$. While no absolutely stable solitons have been found in our numerical simulations, we have observed the effect of soliton revivals after modulational instability is fully developed; see Fig. 3(c). Such an instability can be easily excited if we perturb the stationary localized mode by a small amount $\delta$ as follows: $U(r)[\cos (m \varphi)+i \sin (m \varphi+\delta)]$. The structures that appear at intermediate stages of this instability dynamics are similar to higher-order linear modes such as Laguerre- and Hermite-Gaussian modes. Thus, in this case the nonlocal medium acts as a self-induced mode converter, where the conversion dynamics depends on the initial mode and the properties of the medium, i.e., the value of the nonlocality parameter. After several revivals, the structure loses its symmetry and undergoes irregular dynamics.

In conclusion, we have demonstrated that nonlinear optical media with a nonlocal response can stabilize both stationary and rotating dipole solitons and their generalizations in the form of modulated azimuthons with a fractional spin. The stabilization is achieved when the nonlocality parameter exceeds a certain threshold value, and faster rotating azimuthons are found to be more robust.

S. Lopez-Aguayo is with the Photonics and Mathematical Optics Group, Tecnologico de Monterrey, Mexico. This work was supported by the Australian Research Council. A. S. Destyatnikov's e-mail address is asd124@rsphysse.anu.edu.au.

\section{References}

1. C. Conti, M. Peccianti, and G. Assanto, Phys. Rev. Lett. 92, 113902 (2004).

2. X. Hutsebaut, C. Cambournac, M. Haelterman, A. Adamski, and K. Neyts, Opt. Commun. 233, 211 (2004).

3. C. Rotschild, O. Cohen, O. Manela, M. Segev, and T. Carmon, Phys. Rev. Lett. 95, 213904 (2005).

4. W. Krolikowski and O. Bang, Phys. Rev. E 63, 016610 (2000).

5. W. Krolikowski, O. Bang, and J. J. Rasmussen, Phys. Rev. E 64, 016612 (2001).

6. J. Wyller, W. Krolikowski, O. Bang, and J. J. Rasmussen, Phys. Rev. E 66, 066615 (2002).

7. O. Bang, W. Krolikowski, J. Wyller, and J. J. Rasmussen, Phys. Rev. E 66, 046619 (2002).

8. A. I. Yakimenko, Yu. A. Zaliznyak, and Yu. S. Kivshar, Phys. Rev. E 71, 065603 (2005).

9. D. Briedis, D. E. Petersen, D. Edmundson, W. Krolikowski, and O. Bang, Opt. Express 13, 435 (2005).

10. M. Soljacic and M. Segev, Phys. Rev. Lett. 86, 420 (2001).

11. A. S. Desyatnikov and Yu. S. Kivshar, Phys. Rev. Lett. 88, 053901 (2002).

12. A. S. Desyatnikov, A. A. Sukhoroukov, and Yu. S. Kivshar, Phys. Rev. Lett. 95, 203904 (2005).

13. Yu. S. Kivshar and G. P. Agrawal, Optical Solitons (Academic, 2003), p. 540.

14. A. S. Desyatnikov, Yu. S. Kivshar, and L. Torner, in Progress in Optics, E. Wolf, ed. (North-Holland, 2005), Vol. 47, p. 291, and references therein.

15. T. Carmon, R. Uzdin, C. Pigier, Z. H. Musslimani, M. Segev, and A. Nepomnyashchy, Phys. Rev. Lett. 87, 143901 (2001).

16. V. A. Mironov, A. M. Sergeev, and E. M. Sher, Sov. Phys. Dokl. 26, 861 (1981).

17. B. A. Malomed, in Progress in Optics, E. Wolf, ed. (North-Holland, 2002), Vol. 43, p. 71. 